Cet article a pour objectif

d'expliquer le principe

de fonctionnement du laser,

source lumineuse aux

propriétés d'émission bien

particulières.

Un laser est constitué

d'un milieu matériel dans

lequel I'amplification

de la lumière est possible,

d'un système de pompage

qui fournit de l'énergie

à ce milieu et d'une cavité

optique.

Dans un premier temps,

on décrit le processus

d'émission stimulée à la base

de l'amplification du rayonne-

ment. On présente ensuite

la cavité optique, qui permet

de passer d'un système

qui amplifie la lumière

à une source lumineuse.

Cette cavité impose au faisceau

laser ses caractéristiques

spatiales et temporelles,

qui le distinguent de la lumière

émise par les sources usuelles.

\title{
Le laser : principe de fonctionnement
}

Catherine Schwob $^{(1)}$ (schwob@insp.jussieu.fr) et Lucile Julien ${ }^{(2)}$

(1) Institut des Nanosciences de Paris, UPMC/CNRS, Campus Boucicaut, 140 rue de Lourmel, 75015 Paris (2) Laboratoire Kastler Brossel, ENS/UPMC/CNRS, 24 rue Lhomond, 75231 Paris Cedex 05

\section{Les lasers fêtent leurs 50 ans}

Le premier laser a vu le jour en mai 1960, dans un petit laboratoire industriel à Malibu en Californie. Il s'agissait d'un laser à rubis, fonctionnant en impulsions dans le rouge. Son inventeur, Theodore Maiman, créait ainsi le premier «maser optique ", concrétisant la proposition faite deux ans plus tôt par Arthur Schawlow et Charles Townes de réaliser un oscillateur optique, sur le modèle des masers inventés en 1954 dans le domaine des micro-ondes. Mais passer au domaine optique était loin d'être évident, et de nombreux chercheurs s'y essayaient dans leur laboratoire. Le succès de Maiman fut suivi rapidement de beaucoup d'autres. Le premier laser hélium-néon fut mis au point quelques mois plus tard par Ali Javan aux Bell Labs.

Dans les années suivantes, une grande variété de lasers vit le jour, fonctionnant avec des milieux et des longueurs d'onde de plus en plus diversifiés. De nos jours, cette " curiosité de laboratoire " est devenue un objet courant de la vie quotidienne.

\section{Un faisceau de lumière concentrée et ordonnée}

Un faisceau laser se reconnait du premier coup d'œil, car il est différent de la lumière ordinaire : c'est un faisceau de lumière cohérente, qui se distingue de la lumière émise par les lampes classiques (lampes à incandescence, tubes fluorescents, diodes électroluminescentes). Celles-ci émettent leur lumière dans des directions multiples, ce qui est bien adapté pour éclairer une pièce ou une région de l'espace. Au contraire, le faisceau émis par un laser est un fin pinceau se manifestant, lorsqu'il est arrêté par un obstacle tel qu'un mur, par une tache brillante et presque ponctuelle. Lorsqu'il se propage, même sur de grandes distances, le faisceau laser reste bien parallèle et localisé : cette propriété est la cohérence spatiale.

Une autre caractéristique du faisceau laser, qui apparait dans le domaine visible, est sa couleur bien souvent pure. Dans certains cas, il est quasi monochromatique, dans d'autres il n'est composé que de certaines longueurs d'onde particulières ; cette propriété est la cohérence temporelle.

Ces propriétés de cohérence sont celles du champ électromagnétique émis par le laser. Un tel champ est caractérisé par sa fréquence, sa direction de propagation et sa polarisation. Si l'on traite quantiquement ce champ, c'est-à-dire qu'on le décrit en termes de photons, ces caractéristiques définissent ce qu'on appelle un mode $d u$ champ. Les photons d'un faisceau laser sont donc dans un seul mode du champ ou dans un nombre restreint de modes.

\section{L’amplification stimulée de rayonnement}

Le mot laser, s'il est devenu un terme commun, est à l'origine un acronyme pour "Light amplification by stimulated emission of radiation", c'est-à-dire "Amplification de lumière par émission stimulée de rayonnement ». Comme il apparaît dans cette dénomination, l'émission stimulée - appelée également émission induite - joue un rôle clé dans le fonctionnement des masers et des lasers.

L'émission stimulée est un processus d'interaction entre lumière et matière, comme l'absorption et l'émission spontanée (voir encadré 1). Pour décrire ces interactions, nous prendrons ici l'exemple d'un gaz où les processus d'interaction sont individuels : ils concernent un atome isolé et s'accompagnent de l'apparition ou de la 


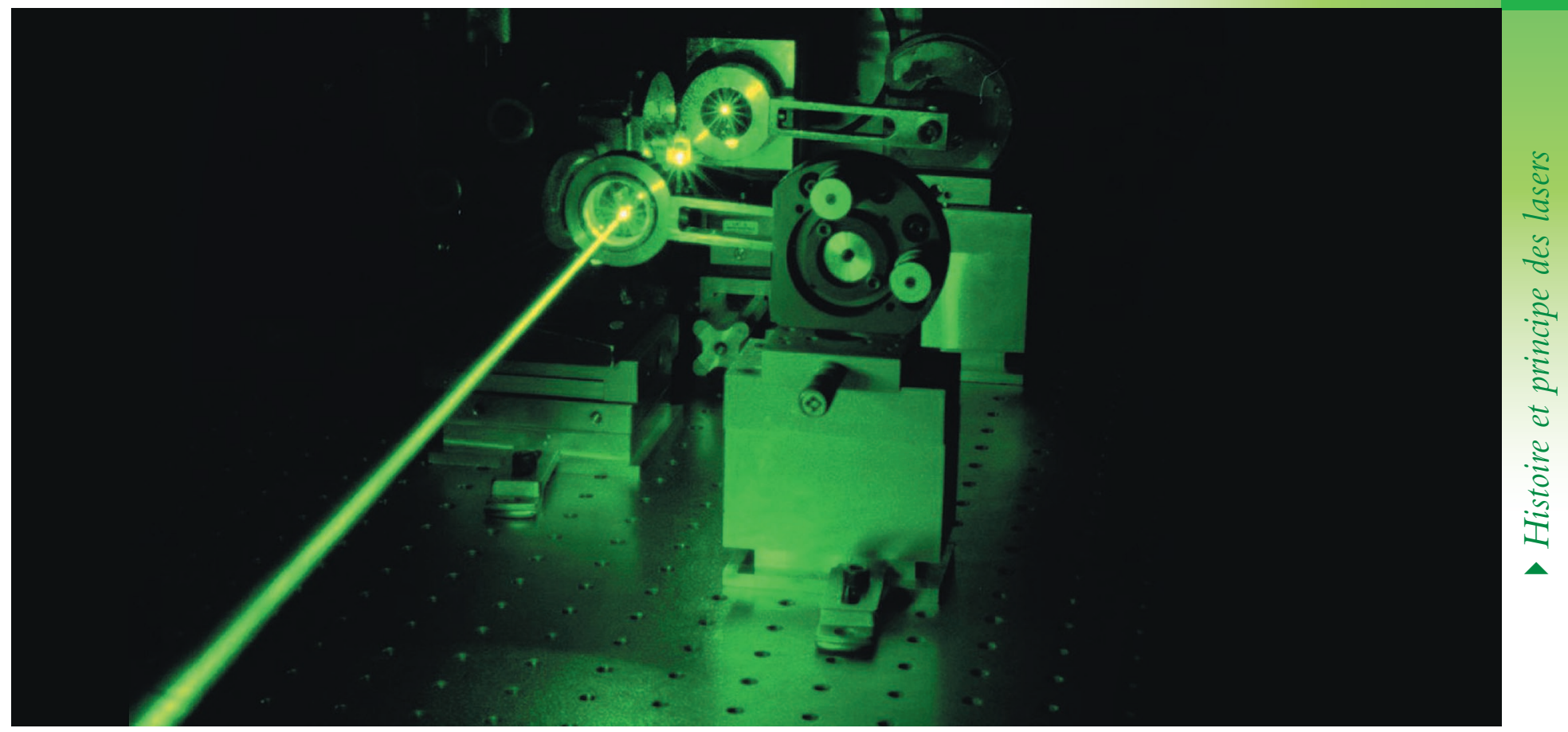

Laser Nd:GdCOB autodoublé, pompé par diode et émettant une puissance de 120 mW vers 530 nm. @ cNRS Photothèque / LCFI0 / GEORGES Patrick.

\section{Les trois processus d'interaction résonante atome-rayonnement}

Plaçons-nous dans le cas de la résonance optique, où les atomes interagissent avec un rayonnement accordé sur une transition atomique, $c^{\prime}$ est-à-dire dont la fréquence $v$ vérifie la relation $E_{2}-E_{1}=h v$.

À l'époque de Bohr, on ne connaissait que deux processus d'interaction, I'absorption et l'émission spontanée. Lors de l'absorption, l'atome passe de son état fondamental d'énergie $E_{1}$, noté 1 , à son état excité d'énergie $E_{2}$, noté 2 , en absorbant un photon (fig. a) ; un photon a disparu de l'onde et celle-ci se trouve atténuée. Lors de l'émission spontanée, l'atome initialement dans son état excité redescend dans son état fondamental en émettant un photon (fig. b). Ce photon est émis dans une direction aléatoire et au bout d'un temps lui aussi aléatoire, mais dont la valeur moyenne est appelée "durée de vie » de l'état excité. L'émission spontanée, comme son nom l'indique, n'a pas besoin de rayonnement incident pour se manifester.

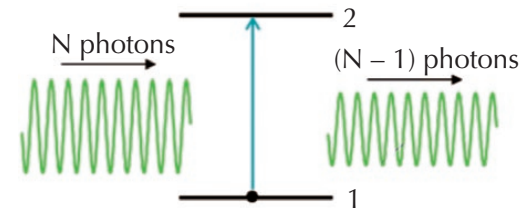

Le processus d'absorption fait disparaitre un photon ; l'absorption atténue l'onde incidente.

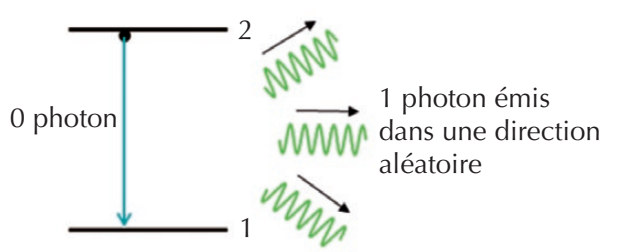

Le processus d'émission spontanée fait apparaître un photon ; la lumière est émise dans une direction et avec une phase aléatoires.
Dans un article publié en 1917, Einstein a introduit un troisième processus, l'émission stimulée. Il s'agit du processus inverse de I'absorption, se produisant, comme elle, en présence de rayonnement incident résonant avec la fréquence de transition. Sous l'effet de celui-ci, l'atome passe de son état excité à son état fondamental en émettant un photon (fig. c). Ce processus est cohérent : si les photons incidents sont dans un mode donné du rayonnement, alors le photon émis l'est dans ce même mode. L'onde émise l'est avec la même direction et la même phase que l'onde incidente : celle-ci se trouve donc amplifiée.

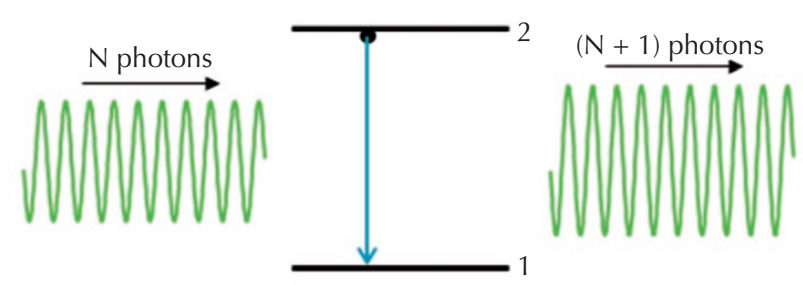

Le processus d'émission stimulée fait apparaître un photon dans le même mode du champ que l'onde incidente ; l'onde est amplifiée. 
〉〉

disparition d'un photon (de plusieurs photons dans le cadre de l'optique non linéaire).

En 1913, Bohr a décrit l'interaction entre un atome et le rayonnement de la façon suivante : l'atome peut absorber ou émettre de la lumière lorsqu'il effectue un "saut quantique " entre deux de ses états d'énergie. Si $E_{1}$ et $E_{2}$ sont les énergies de ces deux états, choisies telles que $E_{2}>E_{1}$, on a la relation $E_{2}-E_{1}=h v$, où $h$ est la constante de Planck et $v$ la fréquence du rayonnement. Le produit $h v$ est l'énergie du photon absorbé ou émis, de sorte que cette relation reflète la conservation de l'énergie dans le processus d'interaction : l'énergie perdue par le rayonnement est fournie à l'atome dans le cas de l'absorption, ou réciproquement dans le cas de l'émission. Il existe trois processus d'interaction entre atomes et rayonnement, décrits dans l'encadré 1. L'émission stimulée introduite par Einstein permet, dans certaines conditions, d'amplifier le rayonnement.

\section{Amplifier la lumière en créant une inversion de population}

Si l'émission stimulée a pour effet d'amplifier la lumière, dans le même temps, l'absorption a pour effet de l'atténuer. Peut-on rendre l'émission stimulée prépondérante?

En pratique, on n'a pas un seul atome en présence du rayonnement, mais un grand nombre d'atomes. Parmi eux, certains sont dans l'état 1, et d'autres dans l'état 2.

Einstein a montré qu'absorption et émission stimulée se produisent avec des probabilités données par la même expression, la seule différence étant que la première est proportionnelle au nombre d'atomes par unité de volume dans l'état 1 (appelé population de l'état 1 et noté $n_{1}$ ), tandis que la seconde l'est à la population $n_{2}$ de l'état excité. Pour que l'émission stimulée l'emporte sur l'absorption, il faut donc que l'on ait $n_{2}>n_{1}$; c'est ce qu'on appelle réaliser une inversion de population.

Cette condition n'est pas facile à obtenir car, laissé à lui-même, un atome se trouve naturellement dans son état fondamental de plus basse énergie, dans lequel l'émission spontanée le ramène toujours. C'est son état d'équilibre.

Pour imposer à l'atome d'être dans un état hors d'équilibre, il faut lui fournir de l'énergie qui le portera dans son état excité afin de réaliser la condition $n_{2}>n_{1}$, soit de façon transitoire, soit de façon permanente. C'est ce que l'on appelle le pompage. Une partie de cette énergie fournie aux atomes sera restituée sous forme de rayonnement à la fréquence $v$ lors de l'amplification. Différentes méthodes de pompage sont possibles : électrique, chimique, optique. De même, des milieux amplificateurs divers peuvent être utilisés : des ions de chrome dans une matrice solide comme c'est le cas pour le laser à rubis, mais aussi, par exemple, des gaz ou des semi-conducteurs, comme détaillé dans l'article de Sébastien Forget et al. (pp. 22-23).

\section{Les éléments constitutifs d'un laser}

Grâce à l'émission stimulée, il est possible par pompage de réaliser une inversion de population, de sorte que les atomes amplifient la lumière. Cependant, un laser est une source de lumière et non pas un amplificateur. Pour réaliser un laser, il faut donc transformer notre amplificateur de lumière en oscillateur.

Une telle transformation est obtenue couramment dans le domaine de l'électronique : en reliant la sortie d'un amplificateur à l'une de ses entrées, le système se met à osciller. C'est aussi elle qui intervient en acoustique dans l'effet Larsen. Dans les deux cas, l'oscillation démarre sur le "bruit" (électrique ou sonore), c'est-à-dire sur des fluctuations de l'environnement. Pour le laser, c'est l'émission spontanée qui jouera le rôle de "bruit".

Pour réaliser un laser, il faut donc renvoyer la lumière dans le milieu amplificateur grâce à un jeu de miroirs, en réalisant une cavité optique. La figure 1 représente le cas d'une cavité en anneau, constituée de quatre miroirs.
La cavité laser la plus simple est constituée de deux miroirs se faisant face. On parle de cavité "Fabry-Perot ", bien connue en interférométrie. Dans une telle cavité, l'un des miroirs réfléchit totalement la lumière à la longueur d'onde considérée. L'autre, le miroir de sortie, transmet une petite fraction de la puissance lumineuse présente dans la cavité ; l'onde transmise constitue le faisceau laser.

La lumière, réfléchie successivement par les deux miroirs, fait des allers-retours dans la cavité. Pour que la lumière vienne, à chaque passage dans l'amplificateur, renforcer l'onde lumineuse qui circule dans le laser, il faut que ces ondes soient en phase. Le chemin optique dans la cavité, correspondant à un aller-retour, doit être égal à un nombre entier de fois la longueur d'onde. C'est la condition de résonance :

$$
2 L=p \lambda \text {, soit } L=p \lambda / 2,
$$

où $L$ est la distance séparant les deux miroirs, $\lambda$ la longueur d'onde de la lumière et $p$ un nombre entier. Pour une longueur $L$ fixée, seules les longueurs d'onde vérifiant la relation ci-dessus pourront donc être présentes dans le faisceau laser.

Les modes associés aux différentes valeurs de $p$ vérifiant cette relation sont appelés modes longitudinaux de la cavité. L'écart en fréquence entre deux modes voisins est donné par $\Delta v=c / 2 L$, où $c$ est la vitesse de la lumière.

En pratique, l'un au moins des miroirs de la cavité doit être concave, afin de concentrer la lumière latéralement pour qu'elle soit recueillie entièrement par les miroirs et limiter ainsi les pertes par diffraction. En conséquence, l'onde lumineuse qui circule dans la cavité laser et le faisceau émis ne sont pas des ondes planes, et le rayon du faisceau n'est pas constant au cours de sa propagation (voir encadré 2).

$\gg>$ 


\section{Col du faisceau laser et longueur de Rayleigh}

Le rayon du faisceau laser prend une valeur minimale, appelée col du faisceau ou "waist" en anglais et usuellement notée $w_{0}$. Cette valeur dépend du rayon de courbure des miroirs de la cavité. Autour de la position correspondant à $w_{0}$, l'onde lumineuse est peu divergente, la taille transverse du faisceau ne varie quasiment pas. À l'opposé, loin du col, l'onde lumineuse peut être assimilée à une onde sphérique : le faisceau est divergent. Dans la cavité laser, c'est généralement autour de la position du col que l'on place le milieu amplificateur, afin d'obtenir le maximum d'émission stimulée, car c'est là que la densité d'énergie est la plus importante (figure ci-dessous).

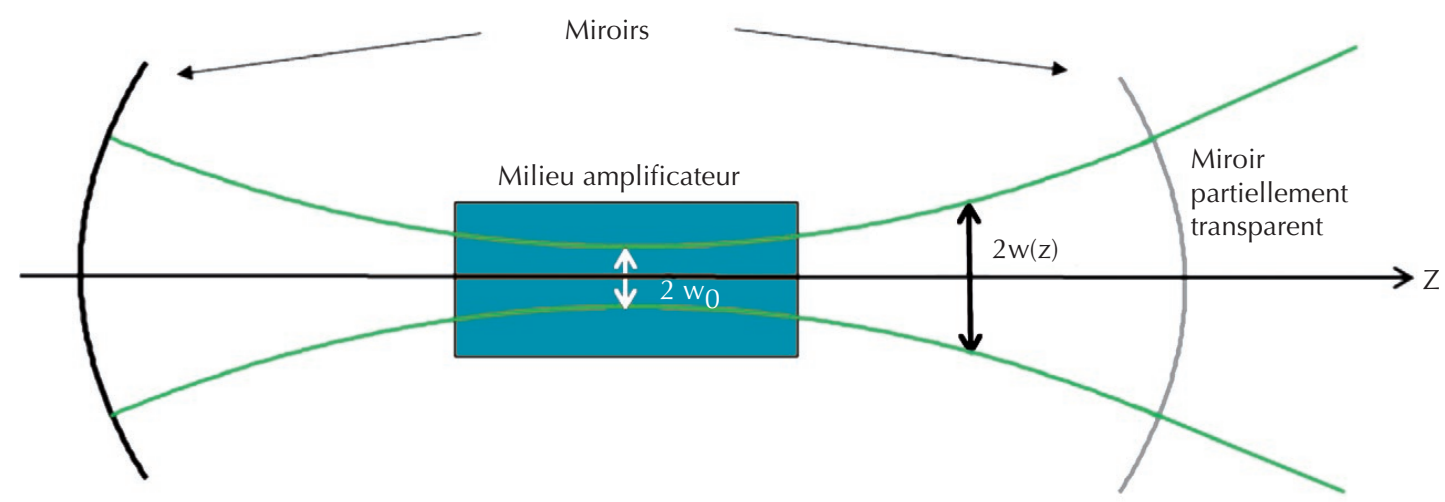

Géométrie du faisceau dans la cavité laser. $w(z)$ est le rayon du faisceau en un point quelconque de sa propagation, $w_{0}$ est le rayon du faisceau au col.

Que ce soit dans la cavité ou à l'extérieur, le rayon du faisceau (noté $w$ ) évolue au cours de la propagation le long de l'axe $z$ suivant la relation $w(z)=w_{0}\left[1+\left(z / z_{R}\right)^{2}\right]^{1 / 2}$. La longueur de Rayleigh, notée $z_{R^{\prime}}$ est la distance à partir du col sur laquelle le rayon du faisceau a augmenté de $w_{0}$ à $(2)^{1 / 2} w_{0}$. Elle est donnée par la relation $z_{R}=\pi w_{0}^{2} / \lambda$ (où $\lambda$ est la longueur d'onde de la lumière), semblable à l'expression de la diffraction de la lumière par un diaphragme de diamètre $2 w_{0}$.

Le faisceau transmis par le miroir de sortie a les mêmes caractéristiques que celles du faisceau incident sur ce miroir. Elles peuvent être adaptées aux besoins de l'utilisateur à l'aide de miroirs ou de lentilles, placées à l'extérieur de la cavité.

Prenons l'exemple d'un laser hélium-néon $(\lambda=633 \mathrm{~nm})$ : si le faisceau est focalisé sur un col de $600 \mu \mathrm{m}$, sa longueur de Rayleigh est d'environ $2 \mathrm{~m}$. Ceci signifie que, sur une distance de propagation de l'ordre de $2 \mathrm{~m}$, le rayon du faisceau reste quasiment constant. Cette propriété du faisceau laser de se propager sur de grandes distances sans diverger est à la base de nombreuses applications ${ }^{(1)}$.

Toutefois, on ne peut pas obtenir simultanément un faisceau très directif et de très petit rayon. En effet, plus on focalise (en utilisant des miroirs concaves de faible rayon de courbure), plus $w_{0}$ est petit, mais plus le faisceau diverge ensuite (la longueur de Rayleigh est également petite).

En pratique, si nécessaire, le faisceau émis par le laser peut être focalisé par une lentille extérieure : le diamètre minimum de la tache qui peut être obtenue ainsi est limité par la diffraction et est de l'ordre de la longueur d'onde. Plus celle-ci est courte, plus la surface nécessaire pour écrire ou lire une information donnée est petite. C'est pourquoi, grâce, entre autres, au développement de lasers à semi-conducteurs de longueurs d'onde de plus en plus petites, la capacité de stockage sur les disques de diamètre $12 \mathrm{~cm}$ a été régulièrement accrue : 650 Mbits pour un CD $(\lambda=780 \mathrm{~nm}), 4,7$ Gbits pour un DVD $(\lambda=650 \mathrm{~nm}), 27$ Gbits pour un "Blue Ray Disk" $(\lambda=405 \mathrm{~nm})$.

(1) L'un des domaines d'application est la télémétrie. Dans le cas de la mesure de la distance Terre-Lune (384 $400 \mathrm{~km}$ ), le faisceau laser a un rayon de l'ordre de $10 \mathrm{~cm}$ et une divergence de 1 rrad à la sortie du télescope, ce qui correspond à une tache de $1 \mathrm{~km}$ de diamètre au niveau de la Lune, si on ne tient pas compte des effets liés à la traversée de l'atmosphère. 
Les éléments constitutifs d'un laser sont donc :

- un milieu amplificateur, pompé dans un état où il peut émettre de la lumière par émission stimulée, et ceci, dans une gamme de fréquences caractéristique du milieu ;

- une source d'énergie assurant le pompage du milieu amplificateur ;

- une cavité optique qui permet le bouclage du dispositif et impose au faisceau émis ses caractéristiques spatiales (direction, divergence) et temporelles (spectre de fréquences)

Une partie de l'énergie lumineuse présente dans la cavité s'en échappe : c'est l'émission du faisceau laser.

\section{Les conditions d'oscillation laser}

Dans un milieu absorbant, l'intensité lumineuse est atténuée lors de la propagation, par un facteur de la forme $\exp (-K l)$, où $K$ est le coefficient d'absorption du milieu, proportionnel à la densité d'absorbant, et $l$ l'épaisseur du milieu traversé.

De la même façon, au sein du laser, le rapport entre l'énergie de l'onde lumineuse après et avant le passage dans le milieu amplificateur, appelé gain de l'amplification, s'écrit : $G=\exp (\alpha l)$, où $\alpha$ est donné par $\alpha=\sigma(v)\left(n_{2}-n_{1}\right)$. La quantité $\sigma(v)$ est la section efficace d'interaction entre les atomes et l'onde ; elle dépend de la fréquence $v$.

En présence d'inversion de population, $n_{2}>n_{1}, \alpha$ est positif et le gain est supérieur à 1 .

Pour que l'oscillation laser démarre, il faut que, pour chaque passage dans le milieu amplificateur, ce gain soit supérieur aux pertes de la cavité : c'est ce que l'on appelle la condition d'oscillation. La principale cause de pertes est la transmission du miroir de sortie. D'autres pertes, que l'on cherche à minimiser, peuvent également exister dans la cavité : absorption, diffusion, réflexion aux interfaces ou diffraction.

En considérant, comme ci-dessus, une cavité formée de deux miroirs, dont l'un seul n'est pas totalement réfléchissant, le gain global sur un tour complet dans la cavité est le produit du gain $G$ et du coefficient de réflexion $R$ de ce miroir. Pour que l'oscillation démarre, il faut que $G \times R \geq 1$. L'égalité donne le seuil d'oscillation. En dessous du seuil, l'intensité de l'onde dans la cavité est négligeable ; au-dessus du seuil, un faisceau laser est émis. Cependant, l'intensité lumineuse dans la cavité, et donc l'intensité émise par le laser à travers son miroir de sortie, ne croissent pas indéfiniment au cours du processus d'amplification. En effet, lorsque l'intensité augmente, des phénomènes de saturation ont pour effet de diminuer le gain de l'amplification. En régime stationnaire, le point de fonctionnement du laser est atteint pour une intensité lumineuse dans la cavité telle que le gain est égal aux pertes.

La condition d'oscillation dépend (i) de la longueur d'onde par l'intermédiaire du gain du milieu amplificateur, l'amplification par émission stimulée n'étant possible que dans la gamme de fréquences caractéristique du milieu, et (ii) des coefficients de réflexion des miroirs. De plus, la cavité optique n'est résonante que pour certaines longueurs d'onde bien particulières, associées aux modes longitudinaux de la cavité. On va donc éventuellement avoir plusieurs modes vérifiant la condition "gain supérieur aux pertes ", chacun pour sa longueur d'onde. On dit dans ce cas que le laser fonctionne en multimode : il émet plusieurs fréquences voisines, séparées de $c / 2 L$. $\mathrm{Si}$, au contraire, un seul mode vérifie la condition, le laser fonctionne en monomode et n'émet qu'une seule fréquence (fig. 2).

Pour certaines applications, il est nécessaire de disposer d'un laser monomode. Pour passer de la configuration multimode à la configuration monomode, on peut sélectionner un mode en insérant à l'intérieur de la cavité laser un élément optique sélectif en fréquence, par exemple une "sous-cavité " plus courte de type FabryPerot (une simple lame de verre peut jouer ce rôle) ; ceci revient à imposer une nouvelle condition de résonance, qui n'est satisfaite que par un seul mode.

\section{Une grande variété de realisations et d'applications}

Depuis la mise au point du premier laser en 1960, des dispositifs lasers de toutes sortes ont été réalisés. Ils utilisent des milieux amplificateurs qui peuvent être gazeux, liquides ou solides, et des méthodes de pompage variées. On trouve en outre de multiples variantes dans la géométrie des cavités. Enfin, leur fonctionnement peut être continu ou en impulsions (relaxé, déclenché ou à modes bloqués) et l'on sait maintenant produire des impulsions femtosecondes $\left(1 \mathrm{fs}=10^{-15} \mathrm{~s}\right)$, de durée comparable à la période de l'oscillation de l'onde électromagnétique.
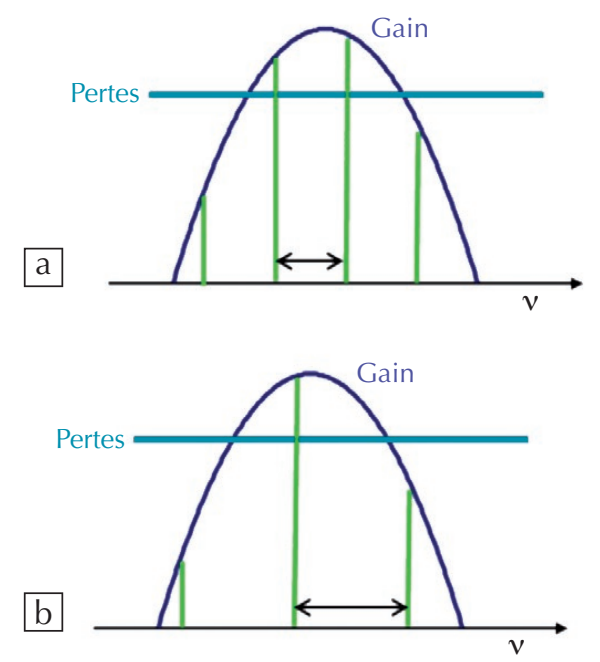

2. Gain du milieu amplificateur en fonction de la fréquence (courbe bleu foncé) et modes de la cavité (traits verts), les pertes du système (ligne turquoise) étant supposées constantes. (a) Si la condition " gain supérieur aux pertes" est vérifiée pour plusieurs modes de la cavité, le laser fonctionne en multimode. (b) Si cette condition n'est vérifiée que pour un seul mode, le laser fonctionne en monomode.

Les propriétés remarquables des faisceaux laser font qu'ils sont utilisés dans de nombreuses applications. Des codes-barres aux imprimantes laser, du stockage à la lecture et au transport d'information, les lasers ont transformé notre vie quotidienne. Dans le bâtiment et l'industrie ils sont des outils universels pour aligner, percer, découper et souder. Ils permettent de mesurer les traces de polluants, les distances et les vitesses. En médecine, ils sont des instruments thérapeutiques sélectifs et précis. Les lasers sont déjà à la base de nombreuses avancées de la recherche fondamentale ; ils seront encore bien présents à l'avenir pour sonder la matière, contrôler la fusion nucléaire, détecter les ondes gravitationnelles, et auront certainement bien d'autres applications que nous ne soupçonnons pas encore... Un grand nombre de ces sujets est traité dans les autres articles de ce numéro spécial.

\section{Pour en savoir plus}

- L. Dettwiller, Les lasers et leurs applications, Ellipses (1998).

- B. Cagnac et J.-P. Faroux, Lasers, Interaction LumièreAtomes, EDP Sciences, collection Savoirs Actuels (2002).

- D. Dangoisse, D. Hennequin et V. Zehnlé, Les Lasers, $2^{\mathrm{e}}$ édition, Dunod (2004).

- C. Delsart, Lasers et Optique Non-linéaire, Ellipses (2008). 